\title{
Ultrathin Au-alloy nanowires: Synthesis and Stability
}

\author{
Dipanwita Chatterjee $^{1}$ and N. Ravishankar*1
}

1. Materials Research Centre, Indian Institute of Science, Bangalore 560012, India

Materials show dramatic changes in properties in the nano regime as compared to bulk. A recent example is that of ultrathin $\left(<2 \mathrm{~nm}\right.$ dia) $\mathrm{Au}$ nanowires ${ }^{1}$ that show significant changes in structure, stability, electron transport, catalytic activity and optical/plasmonic properties compared to bulk $\mathrm{Au}^{2-5}$. An intriguing relaxation mechanism has been observed using transmission electron microscopy (TEM) of ultrathin $\mathrm{Au}$ wires $^{6-8}$. Fundamental aspects of structure and stability can be probed using ultrathin alloy wires; however there is no existing method to synthesize such wires. We have developed a general and consistent template-assisted growth of robust Au-alloy nanowire $(\sim 3.5 \mathrm{~nm}$ diameter $)$ using ultrathin Au nanowires as templates.

The main difficulty associated with the growth of 1-D nanostructures of high-symmetry materials is the symmetry breaking involved in the selection of one orientation over several crystallographically equivalent directions. An oriented attachment mechanism enables the growth of ultrathin Au wires. We use these $\mathrm{Au}$ wires as templates to alloy them using wet chemistry. Since the starting Au wires are very fragile and are not stable even at temperatures as low as $80^{\circ} \mathrm{C}$, it is a challenge to convert these into alloy wires involving diffusion at elevated temperatures. In addition, the wires are also unstable in polar media that lead to removal of surfactant. We have developed a method to convert the Au wires into alloy wires at the liquid-liquid interface. The synthesis involves conversion of $\mathrm{Au}$ nanowire into $\mathrm{AuCu}, \mathrm{AuPd}$ and AuPt nanowire by reduction of the second metal precursor on the template Au nanowires. Fig 1 shows the low magnification TEM images and high-resolution images of the alloy nanowire. An array of wire forms on the grid on drop casting of sample solution. The wires are single crystalline with growth direction of [111]. The formation of the alloy phase has been confirmed by x-ray diffraction analysis and UV-visible spectroscopy as presented and analyzed in Fig 2. The overall composition of the wires as evaluated from TEM-EDS analysis is $\mathrm{Au}-25 \mathrm{at} \% \mathrm{Cu}, \mathrm{Au}-35 \mathrm{at} \% \mathrm{Pt}$ and $\mathrm{Au}-35 \% \mathrm{Pd}$, respectively. STEMEDS mapping also confirms presence of both the elements along the length of the wires.

The alloy nanowires are significantly more stable as compared to the starting Au nanowires. The alloy wires show higher thermal stability and mechanical stability that was impossible to achieve for Au nanowires. The robustness along with the ultrathin dimension of the wire makes these wires ideal candidate for several applications including catalysis and sensing in addition to shedding fundamental insights into the structure, stability and transport behavior.

\section{References:}

(1) Halder, A.; Ravishankar, N. Ultrafine Single-Crystalline Gold Nanowire Arrays by Oriented Attachment. Advanced Materials 2007, 19, 1854-1858.

(2) Chandni, U.; Kundu, P.; Singh, A. K.; Ravishankar, N.; Ghosh, A. Insulating state and breakdown of Fermi liquid description in molecular-scale single-crystalline wires of gold. ACS nano 2011, 5, 8398-8403.

(3) Chandni, U.; Kundu, P.; Kundu, S.; Ravishankar, N.; Ghosh, A. Tunability of electronic states in ultrathin gold nanowires. Advanced Materials 2013, 25, 2486-2491.

(4) Wang, C.; Wei, Y.; Jiang, H.; Sun, S. Bending nanowire growth in solution by mechanical disturbance. Nano letters 2010, 10, 2121-2125.

(5) Pud, S.; Kisner, A.; Heggen, M.; Belaineh, D.; Temirov, R.; Simon, U.; Offenhäusser, A.; Mourzina, Y.; Vitusevich, S. Features of transport in ultrathin gold nanowire structures. Small 2013, 9, 846-852.

(6) Roy, A.; Kundu, S.; Müller, K.; Rosenauer, A.; Singh, S.; Pant, P.; Gururajan, M.; Kumar, P.; Weissmüller, J.; Singh, A. K. Wrinkling of atomic planes in ultrathin Au nanowires. Nano letters 2014, 14, 4859-4866. 
(7) Kundu, P.; Turner, S.; Van Aert, S.; Ravishankar, N.; Van Tendeloo, G. Atomic structure of quantum gold nanowires: quantification of the lattice strain. ACS nano 2013, 8, 599-606.

(8) Yu, Y.; Cui, F.; Sun, J.; Yang, P. Atomic Structure of Ultrathin Gold Nanowires. Nano letters 2016, 16, $3078-3084$.

Acknowledgement: The authors would like to acknowledge DST for funding and AFMM facility of IISc

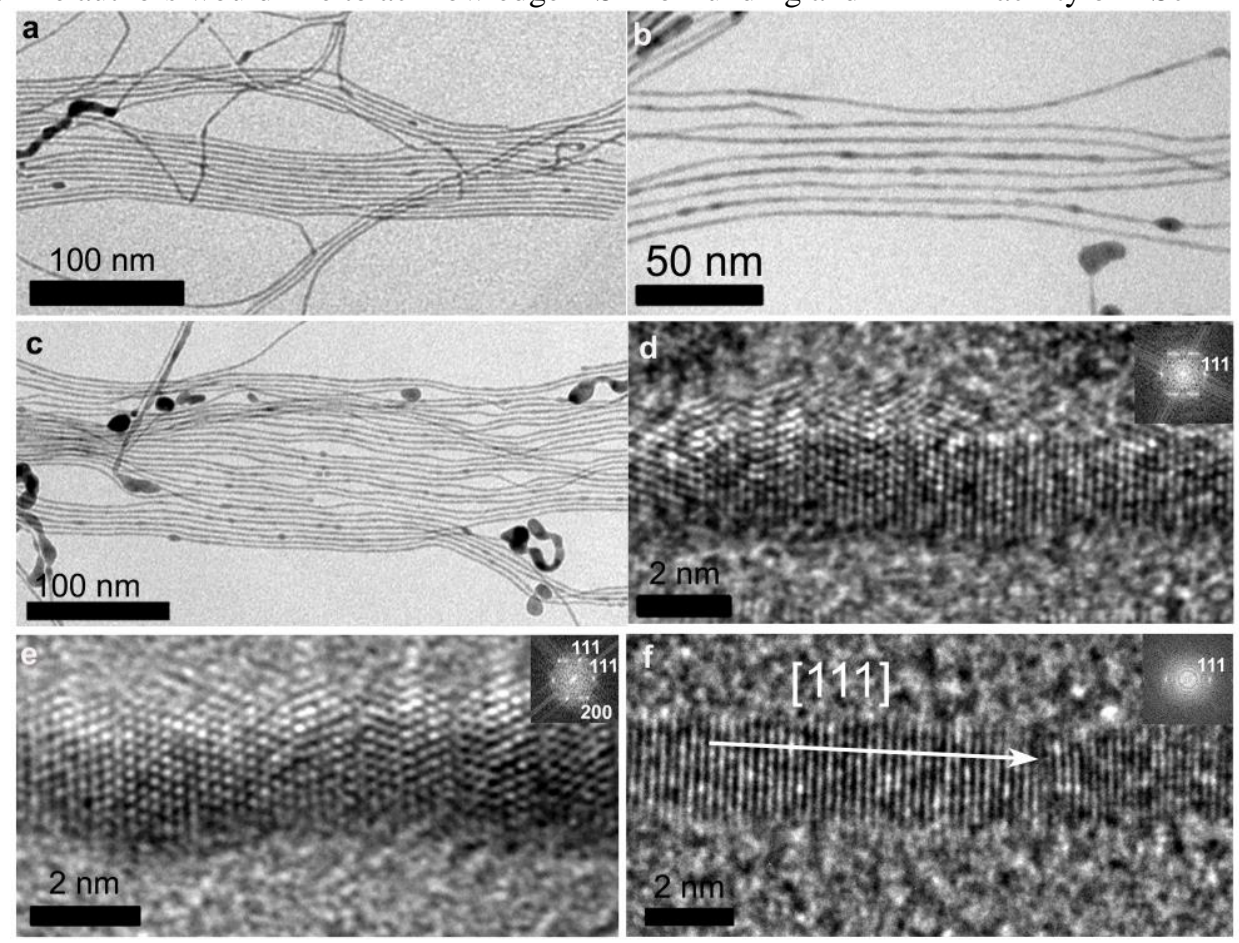

Figure 1. Low magnification TEM images of $\mathrm{AuCu}, \mathrm{AuPd}$ and $\mathrm{AuPt}$ nanowires are shown in $\mathrm{a}, \mathrm{b}$ and $\mathrm{c}$ respectively. High-resolution images of single nanowire of $\mathrm{AuCu}, \mathrm{AuPd}$ and $\mathrm{AuPt}$ are shown in d, e and f respectively. The wires are single crystalline and the growth direction is [111] for all the wires.
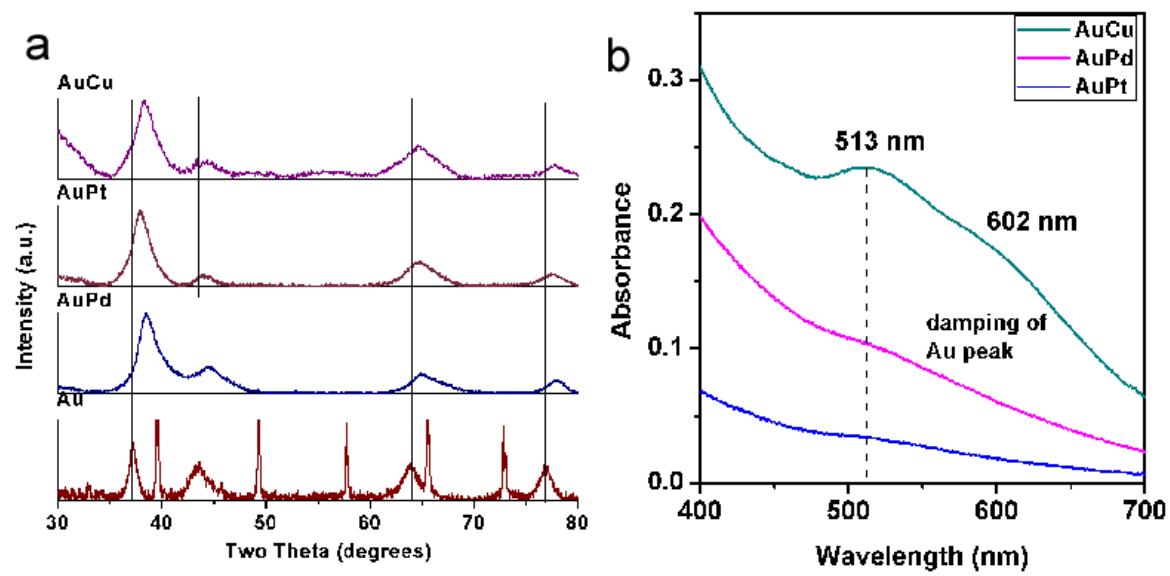

Fig 2. X-Ray diffraction patterns of $\mathrm{Au}, \mathrm{AuPd}, \mathrm{AuPt}$ and $\mathrm{AuCu}$ wires are presented in a. There is a shift of d-spacing of all the alloy wires with respect to pure Au wires indicating mixing of $\mathrm{Au}$ and the second metal. UV-Vis absorbance spectra of the alloy samples are shown in b. Absorbance at $513 \mathrm{~nm}$ and a hump at $602 \mathrm{~nm}$ shows there is pure $\mathrm{Au}$ along with $\mathrm{AuCu}$ wires in the sample. Damping of the peak for $\mathrm{AuPd}$ and AuPt confirms alloying of $\mathrm{Cu}$ with $\mathrm{Au}$. 